\title{
Viscosity of $\left\{x \mathrm{CH}_{4}+(1-x) \mathrm{C}_{3} \mathrm{H}_{8}\right\}$ with $x=0.949$ for Temperatures Between (200 and 423) K and Pressures Between (10 and 31) MPa.
}

Paul L. Stanwix, Clayton R. Locke, Thomas J. Hughes, Michael L. Johns, Anthony R. H.

$$
\text { Goodwin', Kenneth N. Marsh, Eric F. May*. }
$$

Centre for Energy, School of Mechanical \& Chemical Engineering, The University of Western Australia, Crawley, WA, 6009, Australia

${ }^{\dagger}$ also Schlumberger Technology Corporation, Sugar Land, Texas 77478, United States

\begin{abstract}
The viscosity of $\left\{x \mathrm{CH}_{4}+(1-x) \mathrm{C}_{3} \mathrm{H}_{8}\right\}$ with $x=0.949$ was measured at temperatures between (200 and 423) $\mathrm{K}$ and pressures in the range (10 to 31) MPa using a vibrating-wire-viscometer with the wire clamped at both ends and operating in steady-state mode. Over these conditions the fluid mass density, which was calculated from the measured temperature and pressure using the GERG-2008 equation of state, ranged from (120 to 360) $\mathrm{kg} \cdot \mathrm{m}^{-3}$. A three-parameter polynomial in density was able to represent the measured viscosities, which ranged between (19 and 53) $\mu \mathrm{Pa} \cdot \mathrm{s}$, with an r.m.s. deviation of $0.47 \mu \mathrm{Pa} \cdot \mathrm{s}$. This was comparable to the average combined uncertainty of the measurements $(0.43 \mu \mathrm{Pa} \cdot \mathrm{s})$, and no temperature dependence of the viscosity was resolvable within the experimental uncertainty beyond that incorporated within the density obtained from the equation of state. The viscosity of $\mathrm{CH}_{4}+\mathrm{C}_{3} \mathrm{H}_{8}$ reported herein along with measurements previously reported in the archival literature at densities up to $500 \mathrm{~kg} \cdot \mathrm{m}^{-3}$ have
\end{abstract}


been compared with estimates obtained using two corresponding states-type models, which were implemented in the software packages REFPROP and MultiFlash, respectively. The predictions of both models were similar at densities below $200 \mathrm{~kg} \cdot \mathrm{m}^{-3}$, with the extended corresponding states model (ECS) implemented in REFPROP performing better than the SUPERTRAPP model implemented in MultiFlash at higher densities. The relative deviations of the measured viscosities from the ECS REFPROP predictions range from $-5 \%$ around $150 \mathrm{~kg} \cdot \mathrm{m}^{-3}$, which is in the vicinity of methane's critical density, to about $+2 \%$, at the highest densities, whereas the relative deviations from SUPERTRAPP MultiFlash range from $+1 \%$ around $150 \mathrm{~kg} \cdot \mathrm{m}^{-3}$ to -12 $\%$ at the highest densities measured. The trend relative to ECS REFPROP is consistent with those found for literature viscosities measured for mixtures with $x>0.75$.

KEYWORDS: gas viscosity; vibrating wire viscometer

\section{INTRODUCTION}

Thermophysical property measurements of hydrocarbon mixtures with uncertainties fit for the intended purpose of the results can be of critical importance to industry. This importance arises because the semi-empirical models used in process simulation and design often contain unknown and unquantified uncertainties, which are dealt with in practice by over-designing the process with consequential increased capital and operating costs. To achieve better designs that work more effectively and/or over a wider range of conditions, the natural gas industry requires new fundamental property data, both to resolve discrepancies in our current predictive capabilities and to extend them to the higher-pressure conditions characteristic of many new gas fields. Improving this fundamental knowledge will also enable better environmental outcomes through more effective separations (pollutant capture) and more energy efficient processing (reduced 
emissions). However, in general there is presently a dearth of high-quality measurements for hydrocarbon mixtures close to and above their critical point.

In this work, we present measurements of the viscosity of $\left\{x \mathrm{CH}_{4}+(1-x) \mathrm{C}_{3} \mathrm{H}_{8}\right\}$ with $x=0.949$, at temperatures between (200 to 423 ) $\mathrm{K}$ and pressures between (10 to 31) MPa, corresponding to viscosities between (19 and 53) $\mu \mathrm{Pa} \cdot \mathrm{s}$ and densities of (120 to 360$) \mathrm{kg} \cdot \mathrm{m}^{-3}$. This work substantially extends our previous measurements ${ }^{1}$ of viscosity for $x=0.9452$ at pressures between (0.6 and 7) MPa and temperatures of (280 and 298) K, corresponding to densities of (5 to 55) $\mathrm{kg} \cdot \mathrm{m}^{-3}$. The viscosity in both cases was determined with a vibrating-wire-viscometer (VWV), clamped at both ends and operated in steady-state mode, and specifically designed to undertake such measurements of hydrocarbon mixtures. Other workers ${ }^{2-5}$ have reported the viscosity of $\left\{x \mathrm{CH}_{4}+(1-x) \mathrm{C}_{3} \mathrm{H}_{8}\right\}$ for $x$ between $(0.2$ and 0.8$)$ and densities between $(0.5$ and 508) $\mathrm{kg} \cdot \mathrm{m}^{-3}$, obtained with capillary tube, ${ }^{2}$ falling cylinder, ${ }^{3}$ rolling ball, ${ }^{4}$ and oscillating disk ${ }^{5}$ viscometers. We compare our viscosity results to the first three of these sources and their respective deviation from values calculated using two corresponding states-type models, which are the default viscosity models implemented in the software packages REFPROP $9.1^{6}$ and MultiFlash $4.4^{7,8}$ recommended for use by the software supplier. (The data of Abe et al. ${ }^{5}$ were measured only at pressures near atmospheric and were used in the development of the corresponding states models.)

At a given pressure and temperature, knowledge of the fluid mass density is required to determine the viscosity from the resonance curve of the vibrating wire used in this work, and to estimate the viscosity at the measured temperature and pressure from either of the corresponding states models used. In all cases the density was obtained from the GERG- $2008^{9}$ equation of state. However, there are small differences in the implementation of the GERG-2008 EOS between 
software packages and versions: for example, the default implementation of the GERG-2008 EOS in REFPROP 9.1 utilizes more complex equations of state for the pure fluids, with lower uncertainties than those developed originally for the GERG mixture model. In this work we have chosen to use and compare the recommended (default) models for density and viscosity as implemented in REFPROP 9.1 and MultiFlash 4.4 because this reflects the way in which such models are most commonly used. The experimental viscosities reported here were determined from the measured resonance curves using densities calculated using the default implementation of GERG-2008 in REFPROP 9.1.

The recommended models for predicting viscosity in REFPROP 9.1 and MultiFlash 4.4 are different but related. Both are corresponding states models with the SUPERTRAPP ${ }^{10}$ model implemented in MultiFlash being in many respects a forerunner to the Extended Corresponded States (ECS) model $^{11}$ implemented within REFPROP. Accordingly, it is likely that the ECS model will perform as well as or better than the SUPERTRAPP model, and therefore most of the comparisons with data shown here focus on the ECS model in REFPROP, even though in principle the SUPERTRAPP implementation within MultiFlash 4.4 may have been further improved by the software developers. As described by Chichester et al., ${ }^{11}$ the ECS model in REFPROP uses interaction parameters where possible to reduce the difference between the predicted viscosity and values reported in the literature. For the $\mathrm{CH}_{4}+\mathrm{C}_{3} \mathrm{H}_{8}$ system, the binary interaction parameter in the ECS model was obtained using the low-pressure data of Abe et al. ${ }^{5}$ The default values of all binary interaction parameters within all the models applied here were used without modification.

\section{EXPERIMENTAL METHODS}


The vibrating wire viscometer and associated apparatus has been described in detail elsewhere, ${ }^{1}$ so only a brief description will be provided here. The wire was formed from a centerless ground tungsten wire $(\approx 40 \mathrm{~mm}$ long, $50.8 \mu \mathrm{m}$ diameter, with mass fraction purity of 0.9995) clamped and held tautly in a wire holder assembly (formed from stainless steel and polyimide). In the presence of a static magnetic field $(0.45 \mathrm{~T})$, an alternating drive current of between (20 and $80 \mathrm{nA}$ ) excited the mechanical resonance of the wire, which in this case gave a mechanical resonance of about $2 \mathrm{kHz}$ when under vacuum. The amplitude of the wire's motion was monitored by observing the electromagnetically induced voltage, through demodulation and subtraction of the drive signal using a lock-in amplifier; when the drive signal corresponded to the mechanical resonance of the wire it vibrated with a relatively large amplitude, which resulted in a correspondingly large induced voltage. Therefore, under appropriately selected signal conditions, stepping the drive signal frequency through resonance allowed the fluid viscosity to be determined by fitting the measured resonance to the hydrodynamic response function

$$
V_{\text {hydro }}(f)=u(f)-\mathrm{i} v(f)=\frac{\left(A_{1}+\mathrm{i} A_{2}\right) f}{\mathrm{i}\left[f^{2}(1+\beta)-f_{0}^{2}\right]+f^{2}\left(\beta^{\prime}+2 \Delta_{0}\right)},
$$

where the symbols are as defined in equation 2 o Locke et al. ${ }^{1}$ In principle the method provided an absolute measurement of viscosity, ${ }^{12}$ owing to the ability to determine all parameters of the measurement by independent means. In practice, however, the radius of the thin wire used in this work was obtained by calibration with helium, the viscosity of which was determined from ab initio calculations combined with corresponding states theory. ${ }^{1}$

The vibrating wire assembly was housed in a high-pressure vessel, which was evacuated to less than $10 \mathrm{~Pa}$ prior to being filled with the fluid under investigation. In the case of mixtures, the single-phase fluid was transferred from the vessel in which it was prepared gravimetrically into the vibrating wire apparatus. Pressure was controlled using a ISCO model 260D syringe pump 
operated in constant-pressure mode and monitored with an oscillating quartz-crystal transducer (Paroscientific Model 9000-6K-101) having a relative uncertainty of $\pm 0.01 \%$ of its 41 MPa fullscale. Typically, the pressure remained constant during the determination of the resonance curve to within the uncertainty of the transducer, with a relative variation of less than $\pm 0.01 \%$ of the reading. Temperature was controlled by immersing the vessel in a stirred, thermostat bath filled with silicone oil, and the temperature determined with a standard platinum resistance thermometer (Hart Scientific 5699, ITS-90 calibrated), located in the bath in contact with the vessel, of nominal resistance of $25.5 \Omega$ at $T=273 \mathrm{~K}$ with an uncertainty of $\pm 0.005 \mathrm{~K}$. The typical temperature variation about the set-point was $\pm 0.015 \mathrm{~K}$ for integration times of about $60 \mathrm{~s}$ whilst for integration times greater than $0.3 \mathrm{~h}$, which corresponded to the typical frequency sweep time for a single measurement, it was $\pm 0.002 \mathrm{~K}$. Larger temperature variations were experienced at the lowest temperature used in this work of $200 \mathrm{~K}$ (as discussed in more detail later) resulting in approximately three times poorer temperature stability. Pressure, temperature, and the voltage response of the VWV were continuously monitored and logged using custom data acquisition software and saved for subsequent processing and analysis. Further detail about the apparatus and measurement technique are given in our previous work, ${ }^{1}$ and Table 1 shows an analysis of the Type B uncertainty ${ }^{13}$ contributions for the viscosity measurements made here.

The binary mixture $\left\{x \mathrm{CH}_{4}+(1-x) \mathrm{C}_{3} \mathrm{H}_{8}\right\}$ with $x=(0.9490 \pm 0.0001)$ was prepared gravimetrically from pure methane and propane (sources and purities of the mixture components are listed in Table 2). The uncertainty of the mole fraction was determined from contributions arising from the mass measurement uncertainty, mole fraction purity of the component gases, and dead volume in valve atop the mixture preparation cylinder. 


\section{RESULTS AND DISCUSSION}

The measured viscosities for the methane and propane binary mixture are listed in Table 3. Viscosities were determined at each temperature and pressure from between 4 and 156 measurements of the resonance, consisting of between 2 and 78 up-down frequency-sweep pairs. The Type A uncertainties ${ }^{13}$ of the measured viscosities are listed as $u_{i}(\eta)$ in Table 3 , determined from the standard deviation of $v_{\mathrm{i}}$ multiple repeat measurements. For lowest temperature data $(\approx$ $200 \mathrm{~K}$ ) the Type A uncertainties were larger, with a relative value around $0.8 \%$ as compared to less than $0.3 \%$ at higher temperatures. This is attributed to increased instabilities and drift in the temperature of the bath during the associated measurements, even though the bath's PID (Proportional, Integral, and Differential) control parameters were tuned to minimize the temperature instabilities that occurred at $\approx 200 \mathrm{~K}$. The combined uncertainty in the measured viscosities, $u_{\mathrm{C}}(\eta)$, are also listed in Table 3. The combined uncertainties correspond to a coverage factor of $k=1,{ }^{13}$ and were obtained by combining the (Type A) $u_{i}(\eta)$ values in quadrature with the (Type B) $u_{j}(\eta)$ uncertainties listed in Table 1.

The comparison of the measured viscosity data with predictions made at $280 \mathrm{~K}$ (roughly the mid-point of the temperatures studied in this work) using the ECS model in REFPROP and the SUPERTRAPP model in MultiFlash is shown in Figure 1. Three features of this comparison are apparent. First, the predictions of the two models are consistent with each other at densities below $200 \mathrm{~kg} \cdot \mathrm{m}^{-3}$ but differ increasingly at higher densities, with the more recently developed ECS model in REFPROP being more consistent overall with the data measured in this work (relative root mean square deviation (RMSD) of $2.5 \%$ ) than the older SUPERTRAPP model in MultiFlash (7.1\% relative RMSD). Second, as shown in the inset, the ECS model in REFPROP exhibits a small discontinuity of about $0.5 \mu \mathrm{Pa} \cdot \mathrm{s}$ at around $200 \mathrm{~kg} \cdot \mathrm{m}^{-3}$. This feature, which is 
about half the magnitude of the experimental uncertainty of the present data, is caused by the numerical algorithm within REFPROP switching to a slightly different root of the ECS model when determining the physical solution. ${ }^{6}$ Third, the temperature dependence of the measured data is smaller for this mixture than that predicted using the ECS model in REFPROP.

To investigate this third observation further, the following polynomial of density was fit to the viscosity data measured in this work and listed in Table 3.

$$
{ }_{\mathrm{fit}}=a+b^{2}+c^{4}
$$

The three parameters in eq (2) determined by linear least squares regression had the values $a=(18.9 \pm 0.4) \mu \mathrm{Pa} \cdot \mathrm{s}, \quad b=(3.55 \pm 1.28) \times 10^{-5} \mu \mathrm{Pa} \cdot \mathrm{s} \cdot \mathrm{m}^{6} \cdot \mathrm{kg}^{-2}$, and $c=(1.83 \pm 0.09) \times 10^{-9}$ $\mu \mathrm{Pa} \cdot \mathrm{s} \cdot \mathrm{m}^{12} \cdot \mathrm{kg}^{-4}$, the $\mathrm{R}^{2}$ correlation coefficient was 0.998 , and the standard error of the fit was $0.500 \mu \mathrm{Pa} \cdot \mathrm{s}$. These three terms were the most significant for this data set; inclusion of linear or cubic terms did not improve the quality of the fit significantly. The residuals of the fit are shown in Figure 2, plotted in isothermal sub-groups to establish the extent to which the viscosity data contained any further temperature dependence.

No trend with temperature is evident in the residuals $\left(\eta-\eta_{\mathrm{fit}}\right)$, which indicates that for this fixed composition mixture the temperature dependence of the viscosity is the same as that present in the equation of state. Accordingly, once viscosity is expressed as a function of density there is no further variation with temperature to consider. Systematic trends in the deviations are apparent but they are not correlated with temperature and are comparable with the estimated experimental uncertainty. Of course eq (2) and the best-fit parameter values therein are specific to the measurements of a mixture with this composition $(x=0.9490 \pm 0.0001)$, as indicated by 
the small offset apparent in Figure 1 between the viscosities measured at the lowest densities in this work and the viscosities we measured previously ${ }^{1}$ for a mixture with a slightly different composition $(x=0.9452 \pm 0.0002)$. Nevertheless, the regression to eq (2) is useful because (1) it helps establish the consistency and smoothness of our data and the associated uncertainty analysis, and (2) it conveys that less dependence on temperature is observed than that predicted by the ECS model in REFPROP.

While viscosities calculated using the ECS model in REFPROP, $\eta_{\text {calc }}$, have a noticeably different temperature dependence to $\eta_{\mathrm{fit}}$, the slopes in the residuals $\left(\eta-\eta_{\mathrm{fit}}\right)$ with density shown for each isotherm in Figure 2 are similar to the slopes in $\left(\eta_{\text {calc }}-\eta_{\text {fit }}\right)$ with density. This consistency is further apparent for most of the data in Table 3, which shows a direct comparison of the variation with density of all the measured viscosities relative to those calculated using the ECS model in REFPROP. The viscosity data measured previously using this VWV for $x=$ 0.9452 are also shown.

It is apparent from Figure 3 that most of the data measured for $x \mathrm{CH}_{4}+(1-x) \mathrm{C}_{3} \mathrm{H}_{8}$ with $x \approx 0.95$ over a wide range of conditions lie within $\pm 2 \%$ of $\eta_{\text {calc, }}$, which is comparable to the estimated experimental uncertainty, except the region $100<\rho / \mathrm{kg} \cdot \mathrm{m}^{-3}<200$ where the relative deviation in viscosity increases to $-5 \%$. One potential explanation for this increased deviation is the proximity of the mixture density to methane's critical density ${ }^{6} \approx 163 \mathrm{~kg} \cdot \mathrm{m}^{-3}$. This could possibly reflect an increase in the uncertainty of density predictions made using the EOS at these conditions, or alternatively a deficiency in the ECS model; for example, the ECS model does not currently include any form of critical enhancement for viscosity.

Literature viscosity data for the methane + propane binary system reported by Giddings et al. ${ }^{2}$, Huang et al. ${ }^{3}$, and Bicher and $\mathrm{Katz}^{4}$ were compared with the VWV measurement results by 
calculating deviations from the ECS model in REFPROP to assess both data quality and predictive performance. The results of the comparisons are shown, respectively, in Figure 4, Figure 5, and Figure 6 as deviation plots, which help account for the variation in temperature, density and composition between the various measurements.

The highest quality and most relevant data to the current work are the measurements of Giddings et al. ${ }^{2}$ who used a capillary viscometer at temperatures between (311 to 411) K, pressures in the range ( 0.1 to 55.1$) \mathrm{MPa}$ and $x$ in the range (0.22 to 0.79$)$, corresponding to densities in the range $(0.6$ to 506$) \mathrm{kg} \cdot \mathrm{m}^{-3}$. The reported relative uncertainty $(k=1)$ of these measurements was $0.54 \%$, and the relative RMSD of these data from the ECS model in REFPROP is $2.2 \%$. However, the deviations are clearly systematic with composition, ranging from about $-4 \%$ for $x=0.791$ to about $+10 \%$ for $x=0.2207$ and generally becoming more positive with increasing propane mole fraction. For the mixture with the highest methane mole fraction $(x=0.791)$, the variation of the deviations with density follow a similar pattern to our data, exhibiting a peak in the magnitude of the deviations in the vicinity of methane's critical density.

Huang et al. ${ }^{3}$ used a falling cylinder viscometer at temperatures between (153 to 311$) \mathrm{K}$, pressures in the range (3.4 to 34$) \mathrm{MPa}$ and $x$ in the range (0.22 to 0.75 ), corresponding to densities in the range (34 to 645$) \mathrm{kg} \cdot \mathrm{m}^{-3}$. They reported the measurement reproducibility to be $2 \%$, and that on average their data were within $\pm 2 \%$ of those reported by Giddings et al. ${ }^{2}$ The relative RMSD of the Huang et al. ${ }^{3}$ data from the ECS model in REFPROP is $4.4 \%$; however, while the deviations are clearly systematic, the effects of temperature and composition are not as separable as was the case for Giddings et al. ${ }^{2}$ The measurements of Huang et al. were made at temperatures in the range (123 to 311$) \mathrm{K}$, which is significantly lower than the (311 to 411$) \mathrm{K}$ 
range of Giddings et al. ${ }^{2}$ The deviations from the ECS model in REFPROP of the few data reported by Huang et $\mathrm{al}^{3}{ }^{3}$ at densities below $200 \mathrm{~kg} \cdot \mathrm{m}^{-3}$, which are for the mixture with the highest methane content $(x=0.753)$, are consistent with those of the VWV measurements for mixtures with $x \approx 0.95$.

Bicher and $\mathrm{Katz}^{4}$ used a rolling ball viscometer at temperatures between (298 to 498) K, pressures in the range ( 0.1 to 34.4$) \mathrm{MPa}$ and $x$ in the range (0.2 to 0.8$)$, corresponding to densities in the range $(0.5$ to 513$) \mathrm{kg} \cdot \mathrm{m}^{-3}$. Their reported measurement uncertainty was typically $3.2 \%$, with a maximum of $8.1 \%$, and in this context the $6.3 \%$ relative RMSD of their data from the ECS model in REFPROP represents a reasonable level of agreement. While there is a trend of increasingly positive deviations with increasing propane content, it is difficult to generalize any trends in these data or interpret their consistency with the VWV measurements because the sources of the fluid density they used in the analysis of their rolling ball data likely had significant uncertainties.

\section{CONCLUSIONS}

A vibrating wire viscometer was used to measure the viscosity of a $\left\{x \mathrm{CH}_{4}+(1-x) \mathrm{C}_{3} \mathrm{H}_{8}\right\}$ mixture with $x=0.949$ at temperatures from (200 to 423 ) $\mathrm{K}$ and pressures in the range (10 to 31 ) MPa. At this mole fraction, the measured viscosity could be represented nearly within the experimental uncertainty by a simple polynomial in density with no further temperature dependence apparent beyond that incorporated within the equation of state calculation of the density. The data were compared with two corresponding-states type models, implemented in the software packages REFPROP and MultiFlash; these models performed similarly at densities up to $200 \mathrm{~kg} \cdot \mathrm{m}^{-3}$ but the latter model over-predicted the data at higher densities. The extended 
corresponding states model in REFPROP was generally able to represent the measured data within $\pm 2 \%$, except at densities around $150 \mathrm{~kg} \cdot \mathrm{m}^{-3}$, which is near the critical density of methane. Viscosity data in the literature for this binary system exhibited similar trends in their deviations from the ECS model in REFPROP for high methane mole fractions to those observed for the data reported here. These deviations follow different trends as the mixture's propane fraction increases, generally becoming more positive. The new data presented in this work will help in the development of more accurate and wide ranging viscosity models for industrially important mixtures. They also help identify where viscosity data in the literature appear to be deficient and/or conditions at which further measurements are required.

\section{AUTHOR INFORMATION}

Corresponding Author

*Email: eric.may@uwa.edu.au

\section{Author Contributions}

The manuscript was written through contributions of all authors. All authors have given approval to the final version of the manuscript.

\section{Funding Sources}

This work was supported by the Australian Research Council's Linkage Program (LP130101018) and the Gas Processors Association (Project 102). E.F.M. acknowledges 


\title{
Chevron for their support of the research through the Gas Process Engineering
}

endowment.

\section{Notes}

The authors declare no competing financial interests.

\author{
ACKNOWLEDGMENT
}

A.R.H.G wishes to acknowledge Schlumberger for permission to collaborate on this project.

\section{REFERENCES}

(1) Locke, C. R.; Stanwix, P. L.; Hughes, T. J.; Kisselev, A.; Goodwin, A. R. H.; Marsh, K. N.; May, E. F., Improved Methods for Gas Mixture Viscometry Using a Vibrating Wire Clamped at Both Ends. J. Chem. Eng. Data 2014, 59, 1619-1628.

(2) Giddings, J. G.; Kao, J. T. F.; Kobayashi, R., Development of a High-Pressure CapillaryTube Viscometer and Its Application to Methane Propane and their MIxtures in Gaseous and Liquid Regions. J. Chem. Phys. 1966, 45, 578-586.

(3) Huang, E. T. S.; Swift, G. W.; Kurata, F., Viscosities and Densities of Methane-Propane Mixtures at Low Temperatures and High Pressures. AIChE J. 1967, 13, 846-850.

(4) Bicher, L. B.; Katz, D. L., Viscosities of the Methane-Propane System. Ind. Eng. Chem. 1943, 35, 754-761.

(5) Abe, Y.; Kestin, J.; Khalifa, H. E.; Wakeham, W. A., Viscosity and DiffusionCoefficients of Mixtures of 4 Light-Hydrocarbon Gases. Physica A 1978, 93, 155-170.

(6) Lemmon, E. W.; Huber, M. L.; McLinden, M. O. NIST Standard Reference Database 23: Reference Fluid Thermodynamic and Transport Properties-REFPROP, version 9.1; National Institute of Standards and Technology: Gaithersburg, 2013.

(7) MultiFlash, 4.4; InfoChem KBC Advanced Technologies PLC: 2014.

(8) User Guide for Models and Physical Properties; InfoChem/KBC Advanced Technologies PLC: 2014.

(9) Kunz, O.; Wagner, W., The GERG-2008 Wide-Range Equation of State for Natural Gases and Other Mixtures: An Expansion of GERG-2004. J. Chem. Eng. Data 2012, 57, 30323091.

(10) Huber, M. L.; Hanley, H. J. M., The Corresponding-States Principle: Dense Fluids. In Transport Properties of Fluids: Their Correlation, Prediction and Estimation, Millat, J.; Dymond, J. H.; Nieto de Castro, C. A., Eds. Cambridge University Press: 1996; pp 283 - 295.

(11) Chichester, J. C.; Huber, M. L., Documentation and Assessment of the Transport Property Model for Mixtures Implemented in NIST REFPROP (Version 8.0). National Institute of Standards and Technology: Gaithersburg, VA, 2008. 
(12) Goodwin, A. R. H.; Marsh, K. N., An Absolute Viscometer for Liquids: Measurement of the Viscosity of Water at T=298.15 K and p=0.1 MPa. J. Chem. Eng. Data 2011, 56, 167-170.

(13) Taylor, B. N.; Kuyatt, C. E., Guidleines for Evaluating and Expressing the Uncertainty of NIST Measurment Results. 1994 ed.; National Institute of Standards and Technology: Gaithersburg, VA, 1994; Vol. 1297.

Table 1. Contribution to Type B Uncertainty of the Viscosities, $u_{j}(\eta)$, Measured in this Work.

\begin{tabular}{lc}
\hline \multicolumn{1}{c}{ source } & $100 \cdot u_{j}(\eta) / \eta$ \\
\hline wire radius calibration & 0.51 \\
nonlinear motion/out of plane motion & 0.44 \\
density of wire material & 0.09 \\
mixture composition & 0.04 \\
vacuum damping & 0.02 \\
pressure sensor & 0.005 \\
temperature sensor & 0.002 \\
total & 0.68 \\
\hline
\end{tabular}

Table 2. Chemical Sample Sources. Purity was Assumed and no Further Chemical Analysis or Purification was Performed.

\begin{tabular}{ccc}
\hline chemical name & source & manufacturer's purity \\
\hline methane & Coregas & 0.99995 \\
propane & Air Liquide & 0.99995 \\
\hline
\end{tabular}


Table 3. Viscosity $\eta$, Type A Uncertainty $u_{i}(\eta)$, Number of Measurements $v_{\mathrm{i}}$, and Combined Uncertainty $u_{C}(\eta)$ as a Function of Temperature $T$ and Pressure $p$ for $\left\{\left(x \mathrm{CH}_{4}+(1-x) \mathrm{C}_{3} \mathrm{H}_{8}\right\}\right.$, with $x=0.949 .{ }^{\text {a }}$ Density $\rho$ Calculated from the GERG-2008 ${ }^{9}$ EOS Implemented in REFPROP 9.1.6,9

\begin{tabular}{crrrrrr}
\hline$T / \mathrm{K}$ & $p / \mathrm{MPa}$ & $\rho_{\text {EOS }} / \mathrm{kg} \cdot \mathrm{m}^{-3}$ & $\eta / \mu \mathrm{Pa} \cdot \mathrm{s}$ & $u_{i}(\eta) / \mu \mathrm{Pa} \cdot \mathrm{s}$ & $v_{\mathrm{i}}$ & $u_{C}(\eta) / \mu \mathrm{Pa} \cdot \mathrm{s}$ \\
\hline 199.918 & 10.367 & 309.47 & 38.14 & 0.92 & 14 & 0.96 \\
200.599 & 13.841 & 323.60 & 42.36 & 0.73 & 10 & 0.78 \\
202.317 & 17.279 & 332.14 & 44.52 & 0.78 & 26 & 0.84 \\
203.359 & 20.735 & 340.20 & 47.39 & 0.87 & 12 & 0.93 \\
203.235 & 24.182 & 348.68 & 50.08 & 0.81 & 15 & 0.88 \\
203.431 & 25.922 & 352.14 & 51.10 & 0.89 & 17 & 0.96 \\
203.356 & 27.628 & 355.69 & 52.94 & 0.83 & 14 & 0.90 \\
204.187 & 31.114 & 361.05 & 55.49 & 0.83 & 10 & 0.91 \\
255.619 & 20.701 & 249.00 & 27.91 & 0.08 & 18 & 0.21 \\
255.515 & 24.135 & 267.43 & 30.85 & 0.05 & 12 & 0.21 \\
255.586 & 25.902 & 275.09 & 32.21 & 0.05 & 24 & 0.23 \\
255.591 & 27.592 & 281.74 & 33.48 & 0.06 & 15 & 0.24 \\
255.575 & 31.095 & 293.76 & 35.77 & 0.18 & 78 & 0.30 \\
311.121 & 20.946 & 172.01 & 20.61 & 0.28 & 6 & 0.31 \\
311.131 & 24.419 & 194.24 & 22.79 & 0.27 & 8 & 0.31 \\
311.082 & 25.869 & 202.51 & 23.48 & 0.11 & 9 & 0.19 \\
311.129 & 27.584 & 211.44 & 24.53 & 0.16 & 14 & 0.23 \\
311.105 & 31.037 & 227.48 & 26.50 & 0.10 & 4 & 0.20 \\
366.882 & 24.192 & 147.58 & 19.97 & 0.08 & 2 & 0.16 \\
366.894 & 25.926 & 156.48 & 20.64 & 0.06 & 4 & 0.15 \\
366.886 & 27.645 & 164.88 & 21.38 & 0.06 & 11 & 0.16 \\
366.889 & 31.144 & 180.68 & 22.60 & 0.22 & 2 & 0.27 \\
423.625 & 24.191 & 120.32 & 19.44 & 0.10 & 6 & 0.17 \\
423.632 & 25.937 & 127.94 & 19.88 & 0.03 & 6 & 0.14 \\
423.633 & 27.640 & 135.14 & 20.32 & 0.10 & 4 & 0.17 \\
423.633 & 31.097 & 149.01 & 21.36 & 0.05 & 6 & 0.15 \\
\hline & & & & & &
\end{tabular}

${ }^{\text {a }}$ Standard uncertainties $u$ for temperature $T$ and pressure $p$ are $u(T)=0.005 \mathrm{~K}$ and $u(p)=0.004$ $\mathrm{MPa}$. 


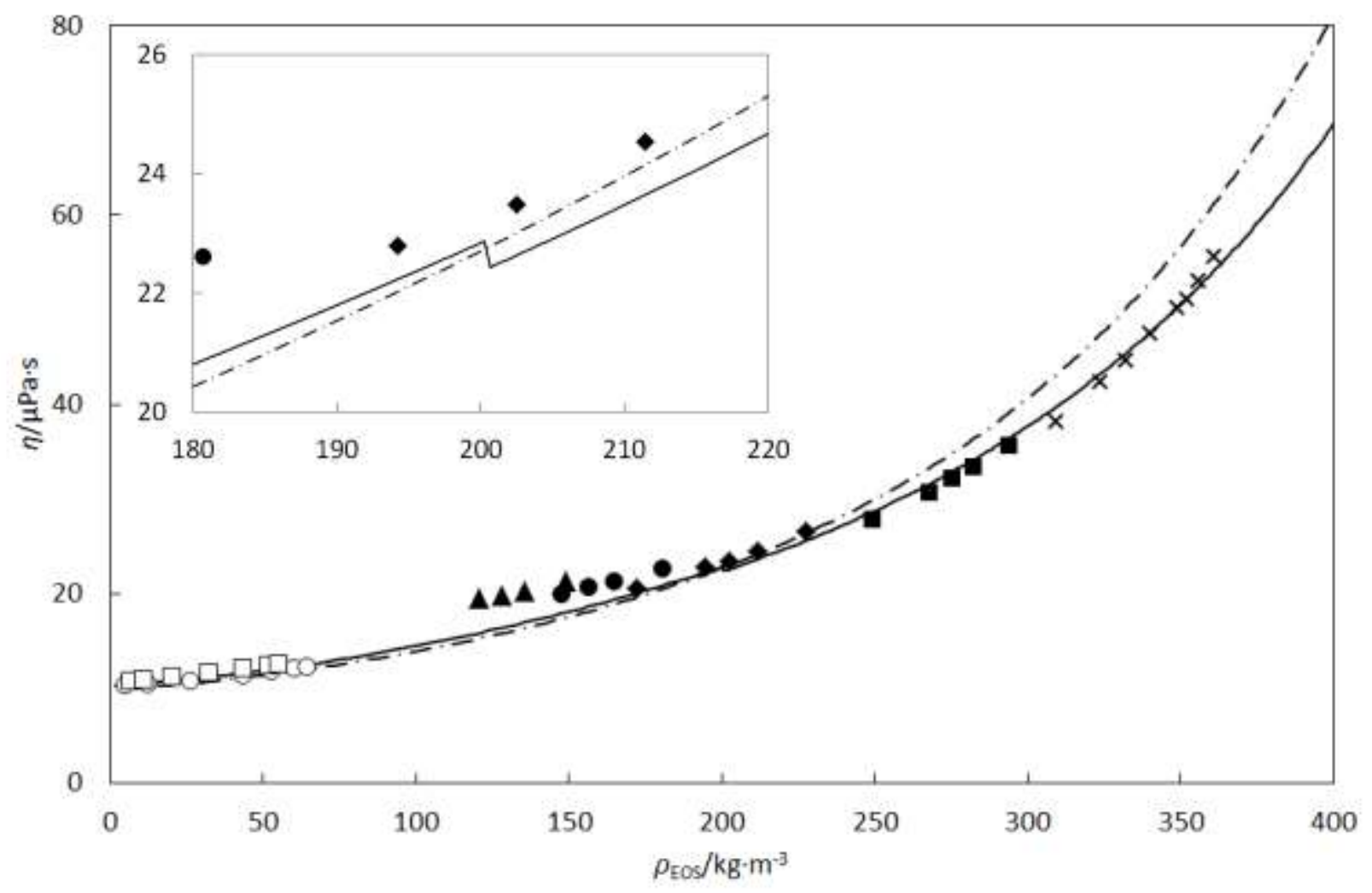

Figure 1. Measured and calculated viscosities $\eta$ for the $\left\{x \mathrm{CH}_{4}+(1-x) \mathrm{C}_{3} \mathrm{H}_{8}\right\}$, with $x=0.949$ system as a function of density $\rho_{\mathrm{EOS}}: \boldsymbol{\Lambda}, T=423 \mathrm{~K} ; \boldsymbol{\bullet}, T=367 \mathrm{~K} ; \bullet, T=311 \mathrm{~K} ; \boldsymbol{\square}, T=256 \mathrm{~K}$; $\times, T=202 \mathrm{~K}$; solid line, predictions of ECS model in REFPROP at $280 \mathrm{~K}$; dash-dotted line, predictions of SUPERTRAPP model in MultiFlash at $280 \mathrm{~K}$. Also shown are our previous measurements with $x=0.9452:^{1} \square, T=280 \mathrm{~K} ; O, T=298 \mathrm{~K}$. Inset shows discontinuity in ECS model predictions at a density of about $200 \mathrm{~kg} \cdot \mathrm{m}^{-3}$. 


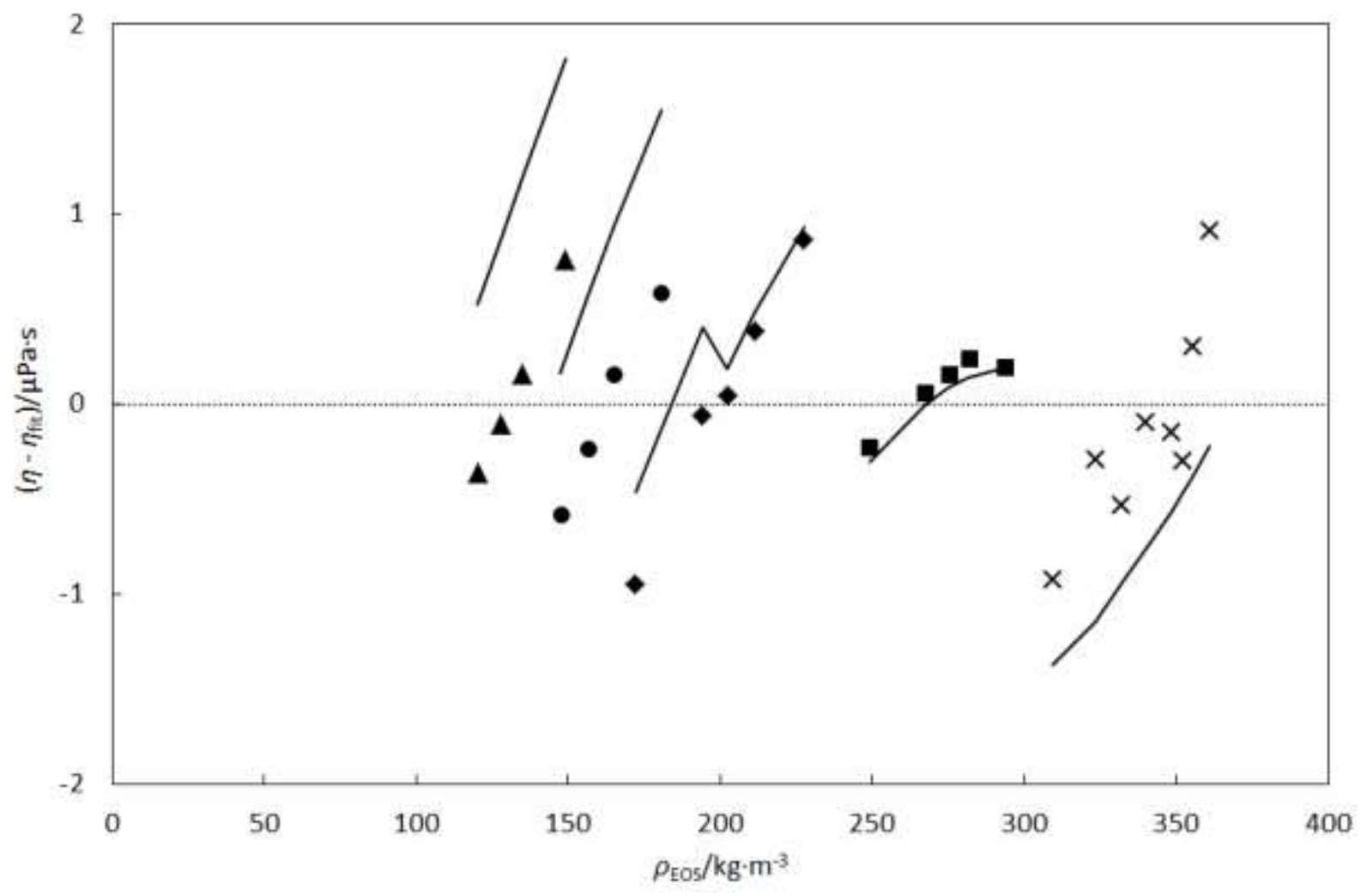

Figure 2. Deviations of the measured viscosity data from the three parameter polynomial in density shown in eq (2), $\eta_{\text {fit }}$, plotted as a function of density $\rho_{\mathrm{EOS}}$ at the five temperatures studied in this work: $\boldsymbol{\Delta}, T=423 \mathrm{~K} ; \boldsymbol{\ominus}, T=367 \mathrm{~K} ; \boldsymbol{\diamond}, T=311 \mathrm{~K} ; \mathbf{\square}, T=256 \mathrm{~K} ; \times \times, T=202 \mathrm{~K}$. While systematic trends in the data residuals are evident there is no trend with temperature apparent and the RMSD is comparable with the experimental uncertainty. Also shown (solid lines) are the differences between predictions made with the ECS model in REFPROP and $\eta_{\text {fit, }}$ which indicate that the ECS model has a much more significant dependence on temperature. 


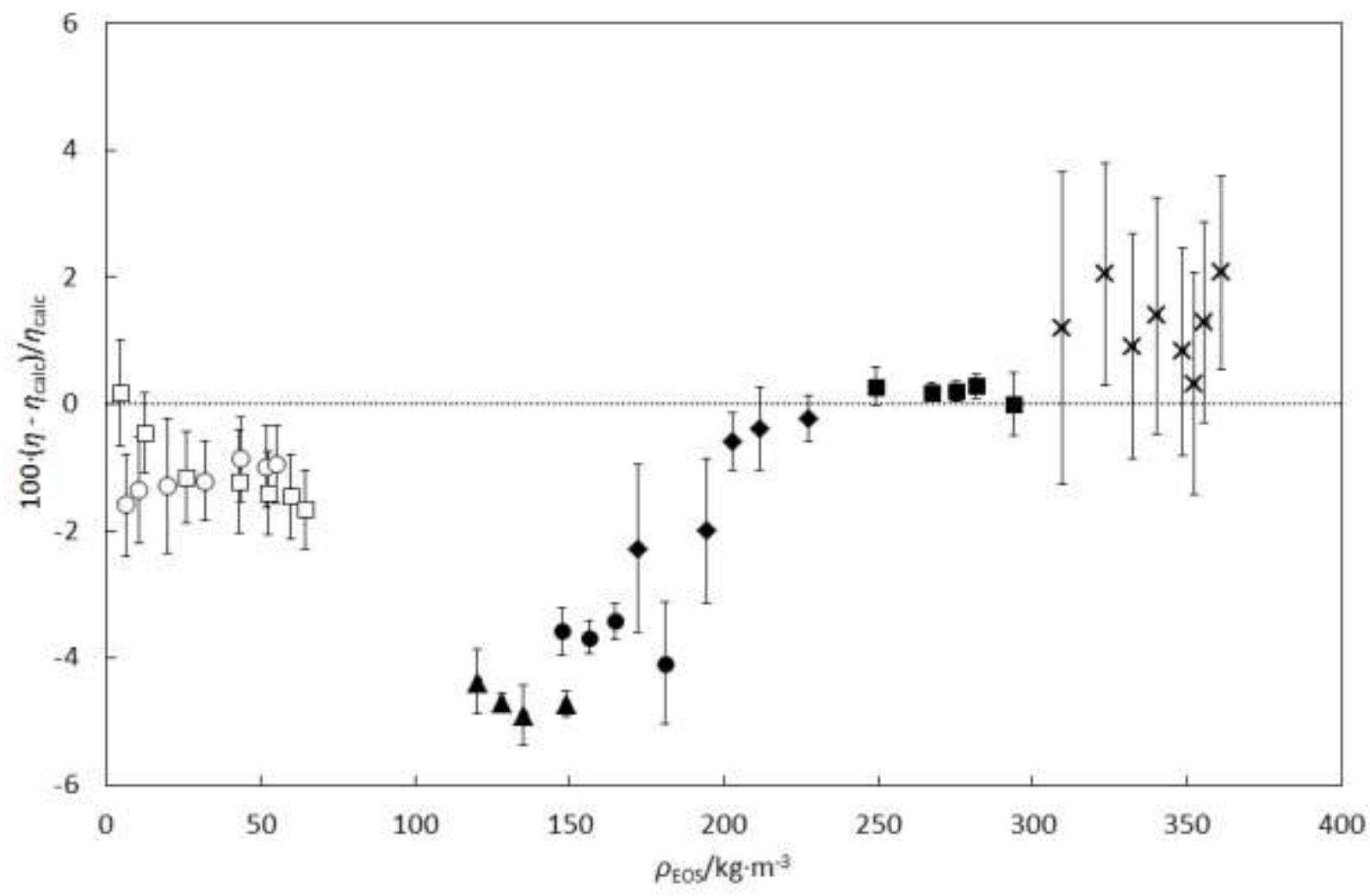

Figure 3. Fractional differences between the measured viscosities $\eta$ and the viscosity calculated $\eta_{\text {calc }}$ using the ECS model in REFPROP for the $\left\{x \mathrm{CH}_{4}+(1-x) \mathrm{C}_{3} \mathrm{H}_{8}\right\}$, with $x=0.949$ system as a function of density $\rho_{\text {EOS }}: \boldsymbol{\Lambda}, T=423 \mathrm{~K} ; \boldsymbol{\bullet}, T=367 \mathrm{~K} ; \boldsymbol{\diamond}, T=311 \mathrm{~K} ; \boldsymbol{\square}, T=256$ $\mathrm{K} ; \times, T=202 \mathrm{~K}$. Also shown are our previous with $x=0.9452:{ }^{1} \square, T=280 \mathrm{~K} ; \mathrm{O}, T=298 \mathrm{~K}$. Error bars represent Type A uncertainty. 


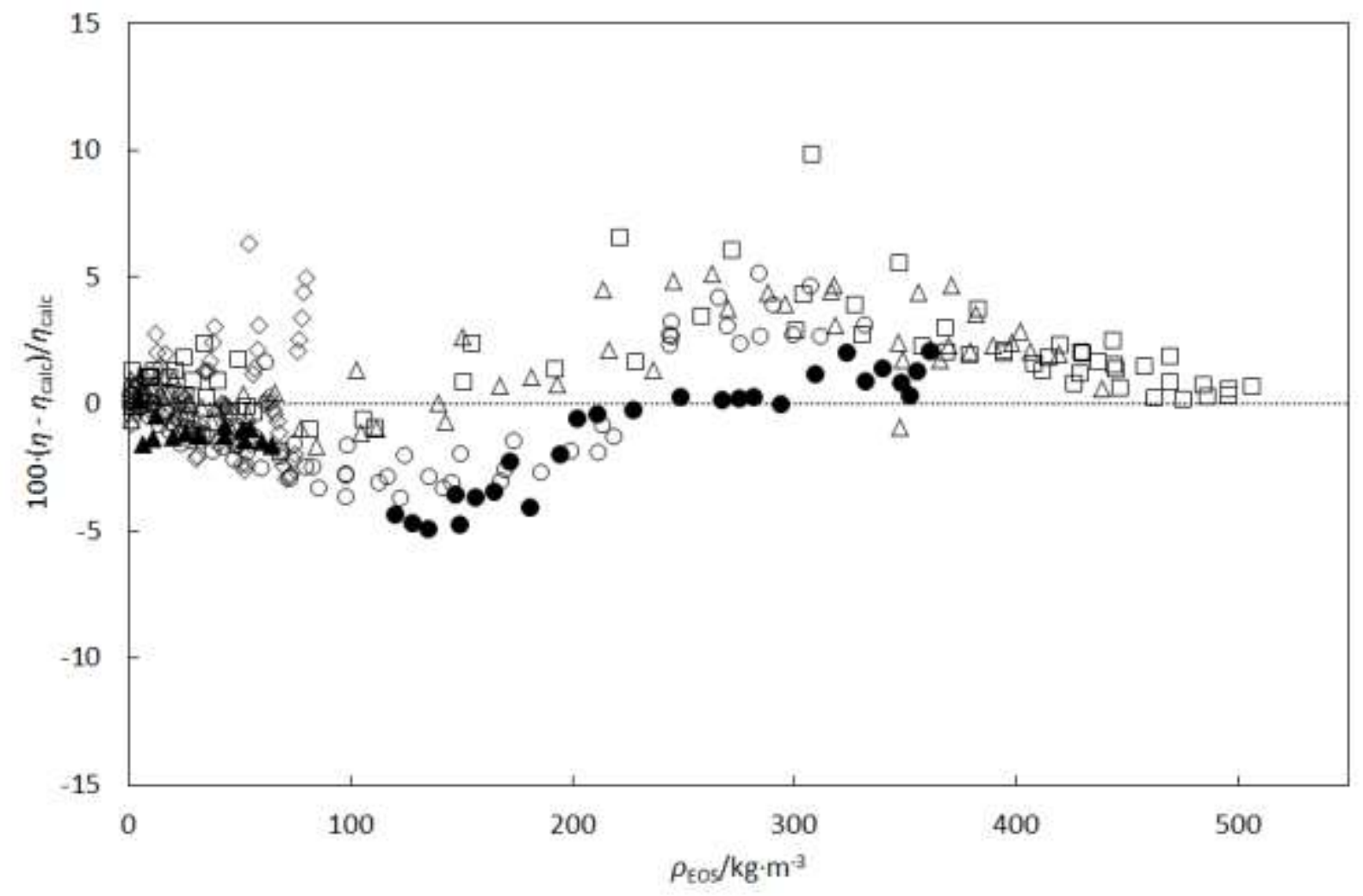

Figure 4. Fractional differences between the measured viscosities $\eta$ and the viscosity calculated $\eta_{\text {calc }}$ using the ECS model in REFPROP for the $\left\{x \mathrm{CH}_{4}+(1-x) \mathrm{C}_{3} \mathrm{H}_{8}\right\}$ system as a function of density $\rho_{\text {EOS. This work: }} \boldsymbol{O}, x=0.949$. Data of Locke et al.: ${ }^{1} \boldsymbol{\Delta}, x=0.9452$. Data of Giddings $e t$ al.: ${ }^{2} \mathrm{O}, x=0.791 ; \diamond, x=0.6139 ; \triangle, x=0.3878 ; \square, x=0.2207$. 


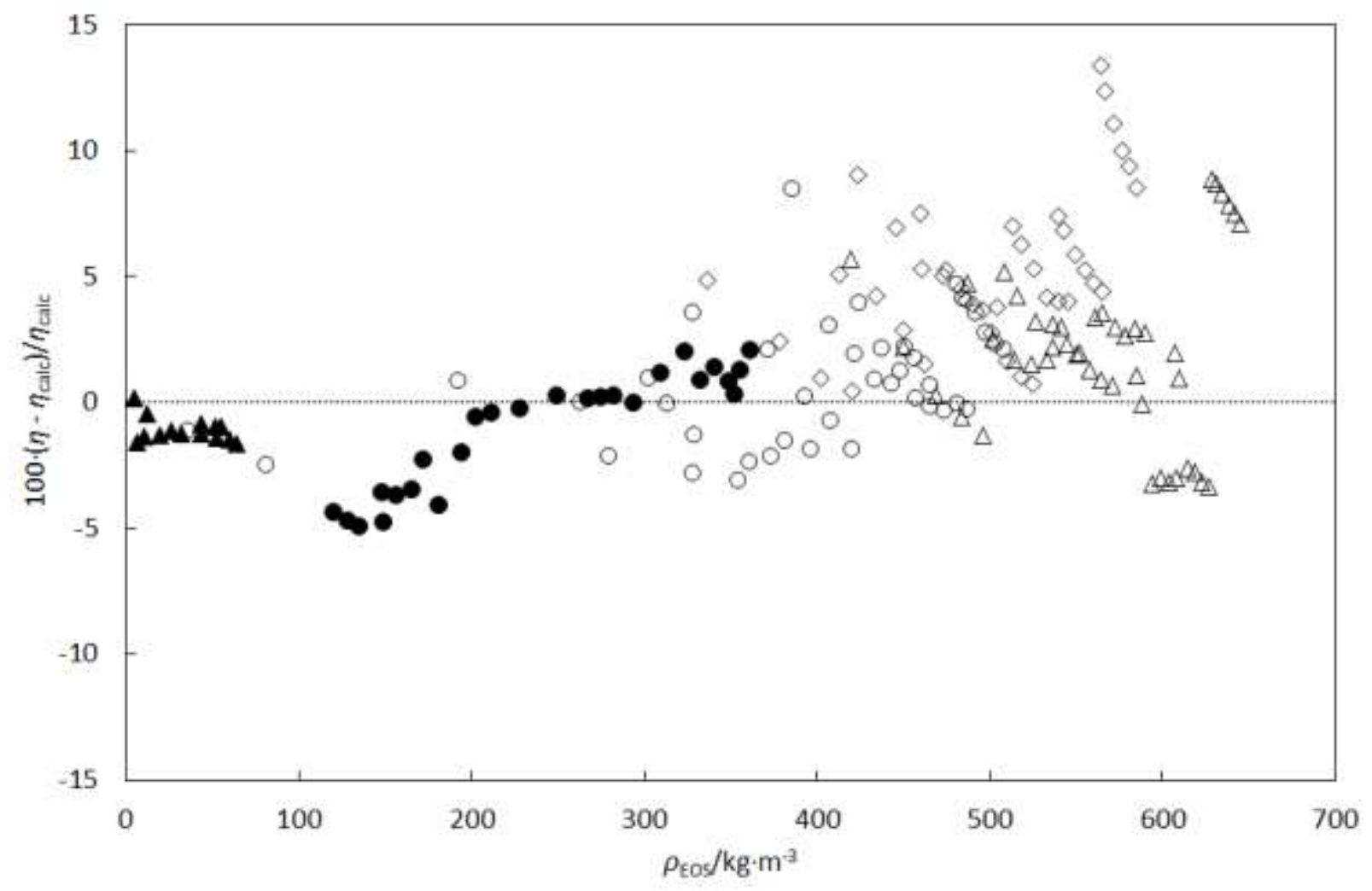

Figure 5. Fractional differences between the measured viscosities $\eta$ and the viscosity calculated $\eta_{\text {calc }}$ using the ECS model in REFPROP for the $\left\{x \mathrm{CH}_{4}+(1-x) \mathrm{C}_{3} \mathrm{H}_{8}\right\}$ system as a function of density $\rho_{\text {EOs. This work: }} \boldsymbol{\bullet}, x=0.949$. Data of Locke et al.: $:^{1} \boldsymbol{\Delta}, x=0.9452$. Data of Huang et $a l .:^{3} \mathrm{O}, x=0.753 ; \diamond, x=0.5 ; \triangle, x=0.221$. 


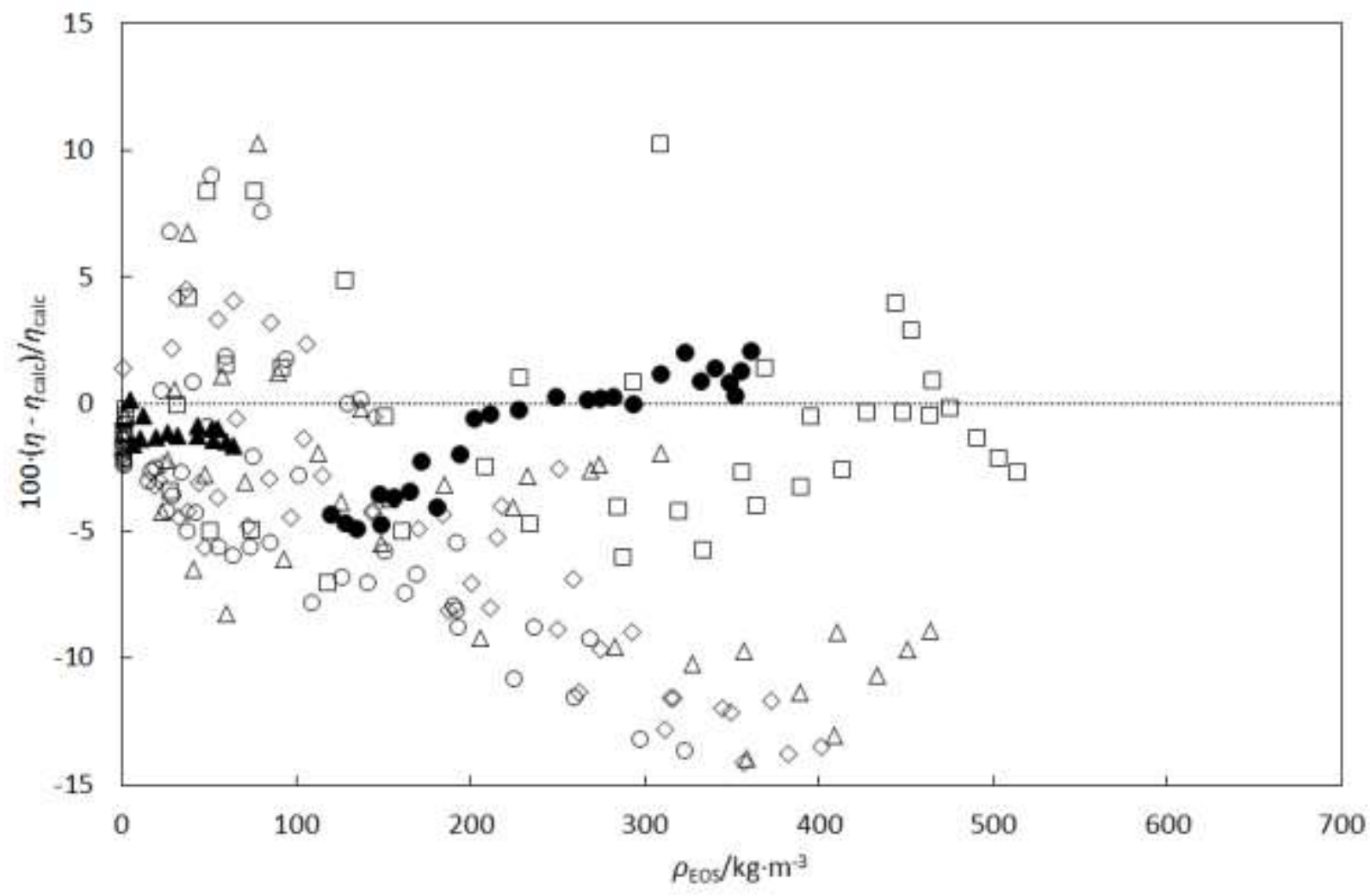

Figure 6. Fractional differences between the measured viscosities $\eta$ and the viscosity calculated $\eta_{\text {calc }}$ using the GERG-ECS model for the $\left\{x \mathrm{CH}_{4}+(1-x) \mathrm{C}_{3} \mathrm{H}_{8}\right\}$ system as a function of density $\rho_{\text {EOs. }}$. This work: $\boldsymbol{\bullet}, x=0.949$. Data of Locke et al. $:^{1} \boldsymbol{\Lambda}, x=0.9452$. Data of Bicher and Katz: ${ }^{4}$ $\mathrm{O}, x=0.8 ; \diamond, x=0.6 ; \triangle, x=0.4 ; \square, x=0.2$. 


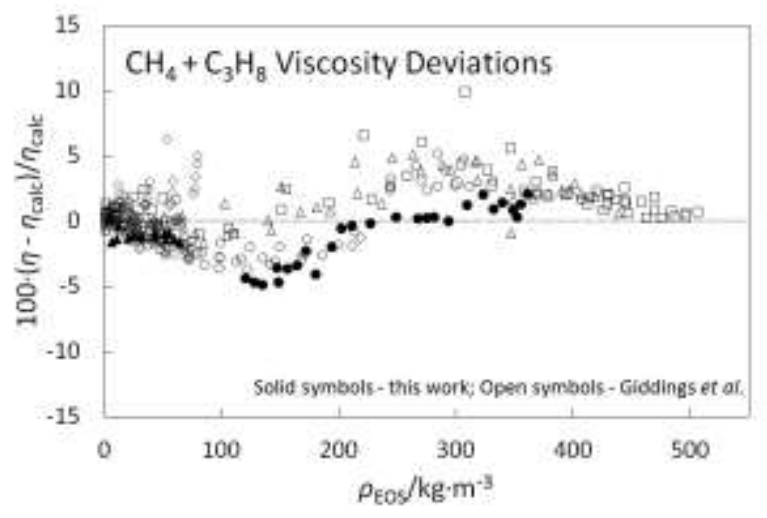

For Table of Contents use only.

Title: Viscosity of $\left\{x \mathrm{CH}_{4}+(1-x) \mathrm{C}_{3} \mathrm{H}_{8}\right\}$ with $x=0.949$ for Temperatures Between (200 and 423) K and Pressures Between (10 and 31) MPa.

Authors: Paul L. Stanwix, Clayton R. Locke, Thomas J. Hughes, Michael L. Johns, Anthony R. H. Goodwin, Kenneth N. Marsh, Eric F. May. 